\title{
A GESTÃO DE INVESTIMENTOS EM TRÊS MANUAIS DE MATEMÁTICA FINANCEIRA: UMA ANÁLISE COMPARATIVA
}

\author{
Ana Carolina Araujo Gonçalves (Universidade Federal do Paraná) \\ anacaro_linas@hotmail.com \\ Maria Eliza C. Lazzaretti (Universidade Federal do Paraná) liza.pugli@ gmail.com \\ Ruth M. Hofmann (Universidade Federal do Paraná) ruthofmann@gmail.com \\ Vitória Assumpção Mendes (Universidade Federal do Paraná) viviam1511 @gmail.com
}

\section{Resumo}

O presente trabalho apresenta uma análise comparativa dos temas contemplados em 3 manuais de matemática financeira que figuram entre os mais vendidos em sites de venda online de livros. Trata-se de uma pesquisa qualitativa e exploratória discutida sob a ótica da literatura sobre a gestão de investimentos na formação em engenharia. Dentre os resultados obtidos pode-se mencionar o fato de que temas basilares como juros, capitalização e sistemas de amortização são contemplados nas três obras, enquanto temas relativamente avançados de gestão financeiras são contemplados em apenas uma. Pode-se mencionar ainda o fato de que os temas elementares contemplados são explicitamente mencionados na Matriz de Conhecimento constante na Resolução 1010/05 CONFEA.

Palavras-Chaves: livro didático; engenharia econômica; ensino em engenharia.

\section{Introdução}

A preocupação com a qualidade do ensino de finanças para Engenharia tem resultado em trabalhos que identificam problemas com métodos de ensino tradicionais, em especial diante dos avanços tecnológicos recentes verificados em sala de aula. Nesse novo cenário, questionase a ênfase concedida pela Engenharia Econômica ao cálculo de indicadores em detrimento do processo de tomada de decisão (EVANS; NACHTMANN; NEEDY, 2010). A formação de competências de gestão econômico-financeira, assim, deve se pautar pelo desenvolvimento de habilidade de análise e de interpretação que não se restringem à matemática financeira.

Para além do domínio de ferramentas matemáticas de avaliação de investimentos, a adequação dos métodos de ensino se soma à delimitação dos conceitos, métodos e técnicas de análise financeira que devem ser contemplados durante a formação de analistas de investimentos. Não obstante deva-se ressaltar o papel dos avanços tecnológicos na redefinição dos meios e das mídias utilizadas no ensino de finanças, os manuais e livros didáticos ainda 
são recursos utilizados por professores e alunos no processo de construção de seu conhecimento financeiro. Assim, conceitos mais recorrentemente abordados durante a formação em análise de investimentos tendem a ser contemplados em livros didáticos utilizados para tal finalidade.

Nesse sentido, a análise de livros didáticos tende a contribuir para a compreensão do perfil do ensino no que se refere à formação de analistas de investimento. É com esse propósito que o presente trabalho apresenta uma análise comparativa dos temas contemplados em 3 manuais de matemática financeira que figuram entre os mais vendidos em sites de venda online de livros. Trata-se de uma pesquisa qualitativa e exploratória discutida sob a ótica da literatura sobre a gestão de investimentos na formação em engenharia.

O artigo está estruturado em quatro seções além desta introdução. A seção 2 apresenta uma breve revisão de literatura sobre o papel, os limites e as perspectivas da gestão de investimentos na formação em Engenharia. A seção 3 apresenta os procedimentos metodológicos do trabalho. A seção 4 contempla os resultados e os discute a partir da literatura. Cabe à última seção apresentar as considerações finais do artigo.

\section{A Gestão de Investimentos na Formação em Engenharia}

Analisando o currículo de 35 cursos de Engenharia Civil, Mecânica, Elétrica e Química, Farr, Merino e Schaefer (2003) constataram que apenas 7 contemplavam Engenharia Econômica. Para os autores, o mercado de trabalho do Engenheiro deve se beneficiar da inclusão da Engenharia Econômica como disciplina obrigatória na formação desse profissional, iniciativa que deve combinar análise econômica e gestão. Trata-se de tarefa não trivial: estudar engenharia econômica requer elasticidade nos estilos de estudo, requer um material de ensino multidisciplinar (ZOGHI, 2015).

Levantamentos acerca dos métodos e técnicas de ensino de finanças para graduação pautamse pela identificação das práticas pedagógicas utilizadas em sala de aula, um esforço de identificar oportunidades de melhorar o ensino de conteúdos econômicos e financeiros (IQBAL; FAROOQI; SAUNDERS, 2006. LELAND, 2017). Tais estudos contemplam, por exemplo, a identificação do uso de projetor e computador em sala de aula, o recurso à discussão de artigos científicos, atividades em grupo, debates e outros métodos de ensino adotados pelo professor (IQBAL; FAROOQI; SAUNDERS, 2006). 
Dentro desse contexto, alguns autores defendem a aplicação da metodologia ativa, ou seja, inverter os papéis de aluno e professor, tornando o discente responsável pelo conhecimento que adquire. Assim, com suporte nessa metodologia, os autores Sanitá, Careta e Zanata (2019) desenvolveram pesquisas enfatizando a importância da utilização de softwares como outro método pedagógico para o desenvolvimento de um raciocínio crítico. $\mathrm{O}$ uso da tecnologia, entretanto, somente se torna eficaz a partir do momento em que o professor deixa de utilizá-la para repassar informações como no ensino tradicional e permite que o estudante se torne o protagonista do seu aprendizado (PEIXOTO, 2016).

Especificamente no que concerne à aplicação de matemática avançada (programação, processos estocásticos, simulação) à análise de investimentos e até mesmo à criação de instrumentos financeiros - principalmente derivativos - a Engenharia Financeira surge como opção para a formação de analistas de investimento (HARTMAN; ENKE, 2007). A Engenharia Financeira, nessa perspectiva, promoveria uma convergência entre finanças, matemática e engenharia, encontrando justamente nas engenharias uma área profícua de desenvolvimento.

Ademais, conhecer os riscos e a viabilidade econômica de um projeto que está sendo desenvolvido é crucial para o sucesso ou fracasso de uma organização e, em termos macroeconômicos, crescimento da economia. Assim sendo, torna-se um assunto de extrema importância para os gestores, que devem estar capacitados para aplicar métodos de análises de investimentos nas empresas (LIZOTE; ANDRADE; SILVA; PEREIRA; PEREIRA, 2014). Considerando-se que a gestão de empresas perpassa inclusive a formação em Engenharia, a gestão de investimentos não pode ser negligenciada nos cursos de graduação de Engenharia.

Para Assaf Neto (1992), tal análise deve se pautar nos seguintes aspectos: fluxo de caixa mínimo, ou seja, a medição da movimentação do caixa em um determinado período; a análise do fluxo de caixa incremental; a taxa mínima de atratividade, isto é, o quanto se espera de retorno para o valor que foi investido; e finalmente, a previsão e análise dos riscos envolvidos. Esse último item é destacado por Pinto et al. (2019, p. 1): “Ao se pensar em investimento, não se pode esquecer de levar em conta os riscos. Eles aparecem sob duas posições: a das possibilidades de rendimentos ou de retornos e as possibilidades de perdas. Portanto, viabilidade econômica de projetos é a tarefa de analisar as expectativas de retorno do investidor associadas aos riscos que determinado investimento pode representar, em relação às variáveis mercadológicas externas". 
Conforme a matemática financeira se torna cada vez mais essencial não apenas na formação em cursos de graduação, mas também no cotidiano, seu ensino passa a ser necessário na formação de cada indivíduo. A aplicação do conhecimento mencionado previamente, portanto, auxilia no entendimento de conceitos como juros, empréstimos e financiamentos, assim, os alunos conseguem ministrar com maior facilidade suas finanças (MIRANDA; PHILIPPSEN, 2014). Tais conceitos compõem os fundamentos da matemática financeira.

Especificamente no que se refere aos cursos de graduação em Engenharia de Produção que operam no Brasil, deve-se ressaltar a existência de regulamentações que impõem obrigatoriedade de oferta de disciplinas de finanças consideradas essenciais à formação de engenheiros. A própria matriz de conhecimentos (ABEPRO, 2020) elenca, no contexto da Engenharia Econômica, um conjunto de conteúdos específicos que incluem, por exemplo, os que constam no quadro 1:

Quadro 1 - Exemplos de temas financeiros constantes na Matriz de Conhecimentos da Engenharia de Produção

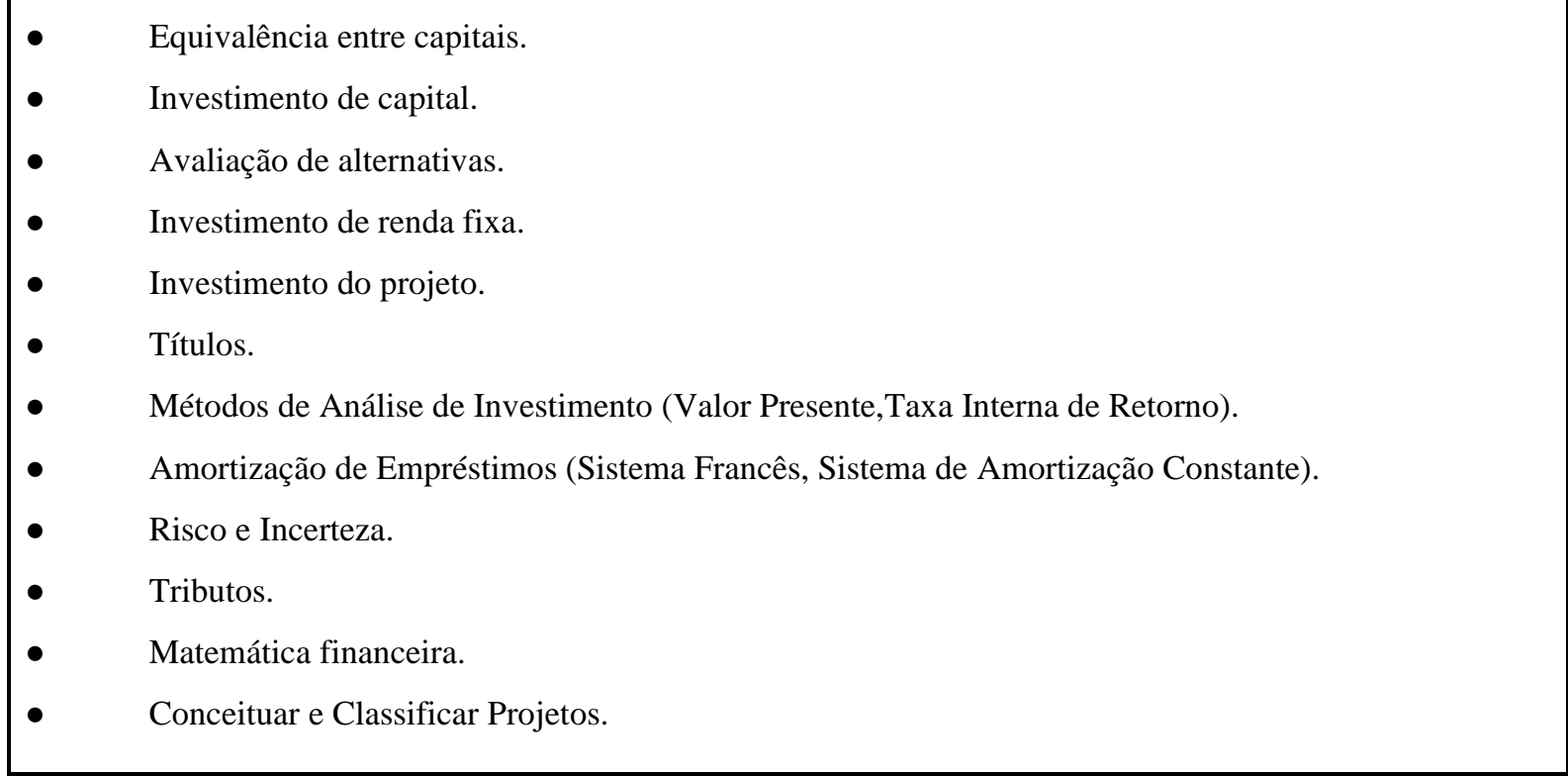

Fonte: Elaborado a partir de ABEPRO (2020)

Note-se que tais conteúdos são lecionados a partir de diferentes abordagens didáticas. Métodos tradicionais de ensino recorrem ao livro didático como instrumento de ensino e que sua importância se expressa, inclusive, pela obrigatoriedade de indicação de bibliografia básica e complementar nas ementas de disciplinas de cursos de graduação no Brasil. É nesse contexto que este trabalho se propõe analisar os temas contemplados nos livros didáticos de matemática financeira. Os procedimentos metodológicos adotados para tanto constam na seção a seguir. 


\section{Metodologia}

Este trabalho se caracteriza como pesquisa exploratória essencialmente qualitativa, tendo-se recorrido à pesquisa documental para a coleta de dados e bibliográfica para proporcionar o contexto da abordagem.

Quantos aos procedimentos metodológicos empregados, procedeu-se inicialmente a identificação dos principais sites nacionais de venda de livros online. Nesse sentido, cabe destacar que o trabalho se restringiu à identificação dos livros mais vendidos em canais online, o que significa que pode haver eventual divergência em relação às vendas em lojas físicas (informações não coletadas para esta pesquisa).

Identificados os principais sites de vendas, foram selecionados os cinco principais para que se passasse às buscas referentes aos livros mais vendidos em Matemática Financeira, sendo esta a expressão utilizada nas buscas de tais sites. A partir dos resultados obtidos para essa busca, aplicou-se o filtro "Mais vendidos" ou equivalente, a depender da estrutura do site em questão.

As pesquisas permitiram identificar os cinco livros de Matemática Financeira mais vendidos em cada site. Os resultados foram tabulados em planilha eletrônica e os três livros mais frequentes em cada um dos sites foram selecionados para comparação. Considerando-se que o propósito do trabalho consiste na comparação dos temas abordados (não foram analisadas variáveis como abordagem didática, quantidade de exercícios disponíveis ou qualquer outro aspecto potencialmente relevante), uma vez identificadas as obras mais vendidas, procedeu-se a coleta de dados dos sumários.

Para este trabalho considerou-se que o sumário dos livros seria uma aproximação robusta do que seriam os temas de gestão financeira contemplados nas obras. Assim, cabe mencionar que a abordagem metodológica selecionada neste trabalho conta com limitações, inclusive diante do fato de que temas específicos podem ser abordados nos livros no formato de exemplos, de exercícios ou de quadros explicativos não constantes explicitamente nos sumários.

\section{Resultados e discussão}

As obras mais vendidas que se repetiram entre as cinco primeiras posições de todos os sites pesquisados são apresentadas no quadro 1: 
Quadro 2 - Livros identificados como "mais vendidos" e selecionados para comparação

\begin{tabular}{|lccc|}
\hline Título & $\begin{array}{c}\text { Matemática Financeira e } \\
\text { suas Aplicações }\end{array}$ & Matemática Financeira & $\begin{array}{c}\text { Matemática Financeira - Juros, Capitalização } \\
\text { simples e composta, Sistemas de amortização } \\
\text { Price e SAC, Títulos públicos: LTN, NTN e } \\
\text { LFT, Taxas de Selic e CDI, Utilização de } \\
\text { calculadoras financeiras }\end{array}$ \\
\hline Autor & Alexandre Assaf Neto & Carlos Patrício Samanez & José Dultra Vieira Sobrinho \\
\hline Edição & 12 & 5 & 8 \\
\hline Ano & 2012 & 2010 & 2018 \\
\hline Editora & Atlas S.A & Pearson & Atlas Ltda \\
\hline
\end{tabular}

Fonte: Os autores (2020)

A partir da análise dos dados foi possível identificar semelhanças e diferenças em termos dos conteúdos listados nos três sumários. As principais semelhanças foram identificadas no que se refere ao fato de os conceitos fundamentais de matemática financeira figurarem no sumário dos três livros comparados. Temas como juros simples e compostos, capitalização e sistemas de amortização, todos constantes na Matriz de Conhecimentos da Engenharia de Produção (ABEPRO, 2020), fazem parte explicitamente do sumário das obras comparadas. Trata-se de um resultado esperado considerando-se serem esses temas essenciais e elementares de matemática financeira (ASSAF NETO, 2012. FARR; MERINO; SCHAEFER, 2003).

As principais diferenças foram identificadas no que se refere a temas mais específicos de análise de investimentos que se inserem no contexto da gestão financeira. Temas que eventualmente possam ser considerados mais avançados (como avaliação de ações, por exemplo), foram identificados pontualmente, não constando no sumário das três obras comparadas. O quadro 3 apresenta a síntese da comparação dos temas abordados nos sumários das obras comparadas.

Quadro 3 - Comparação entre os temas de gestão de investimentos constantes nas obras de matemática financeira selecionadas

\begin{tabular}{|l|c|c|c|}
\hline & $\begin{array}{c}\text { Assaf } \\
\text { Neto } \\
\mathbf{( 2 0 1 2})\end{array}$ & $\begin{array}{c}\text { Vieira } \\
\text { Sobri- } \\
\text { nho } \\
\mathbf{( 2 0 1 8})\end{array}$ & $\begin{array}{c}\text { Sama- } \\
\text { nez } \\
\mathbf{( 2 0 1 0}\end{array}$ \\
\hline Conceitos gerais ou fundamentais, incluindo juros simples & $\mathrm{X}$ & $\mathrm{X}$ & $\mathrm{X}$ \\
\hline Juros compostos & $\mathrm{X}$ & $\mathrm{X}$ & $\mathrm{X}$ \\
\hline Descontos & $\mathrm{X}$ & $\mathrm{X}$ & $\mathrm{X}$ \\
\hline Inflação & $\mathrm{X}$ & $\mathrm{X}$ & $\mathrm{X}$ \\
\hline Caderneta de poupança & $\mathrm{X}$ & & \\
\hline
\end{tabular}




\begin{tabular}{|l|c|c|c|}
\hline Duplicatas & $\mathrm{X}$ & $\mathrm{X}$ & \\
\hline Commercialpapers & $\mathrm{X}$ & $\mathrm{X}$ \\
\hline Cálculo do custo efetivo & $\mathrm{X}$ & $\mathrm{X}$ & $\mathrm{X}$ \\
\hline Factoring & $\mathrm{X}$ & & $\mathrm{X}$ \\
\hline Hot Money & & & $\mathrm{X}$ \\
\hline Export note & & & $\mathrm{X}$ \\
\hline Conta garantida & $\mathrm{X}$ & & $\mathrm{X}$ \\
\hline Taxas Over & & $\mathrm{X}$ & $\mathrm{X}$ \\
\hline Fluxos de Caixa & $\mathrm{X}$ & $\mathrm{X}$ & $\mathrm{X}$ \\
\hline Análise de Investimentos e Reposição de Ativos & $\mathrm{X}$ & $\mathrm{X}$ & $\mathrm{X}$ \\
\hline Sistemas de Amortização de Empréstimos e Financiamentos & $\mathrm{X}$ & $\mathrm{X}$ & $\mathrm{X}$ \\
\hline Taxa e Prazo Médio de Operações Financeiras & $\mathrm{X}$ & $\mathrm{X}$ & \\
\hline Títulos Públicos & $\mathrm{X}$ & $\mathrm{X}$ & $\mathrm{X}$ \\
\hline Títulos de Renda Fixa & $\mathrm{X}$ & & \\
\hline Avaliação de Ações & & \\
\hline
\end{tabular}

Fonte: Elaborado pelos autores a partir dos dados coletados (2020)

Cabe mencionar que na obra de Assaf Neto (ano) o sumário contém tópicos detalhados para o tema Títulos Públicos, incluindo desde conceitos como a Taxa Selic até aspectos metodológicos e operacionais, tal como consta no quadro 4:

Quadro 4 - Exemplo de detalhamento de "Títulos Públicos” no sumário da obra de Assaf Neto

\begin{tabular}{|ll|}
\hline Taxa SELIC & Cálculo do valor de mercado da LFT mercado \\
Preço unitário (PU) de um ativo & secundário \\
Contratos futuros de juros & Notas do Tesouro Nacional Série F (NTN-F) \\
Marcação a Mercado (MaM) & Cálculo do PU de uma NTN-F \\
Principais medidas dos títulos públicos & Operação de leilão primário de NTN-F \\
Letras do Tesouro Nacional (LTN) & Notas do Tesouro Nacional Série B (NTN-B) e Série \\
Exemplos: leilão primário de LTN & C (NTN -C) \\
Exemplos: mercado secundário de LTN & Negociação de NTN-B no mercado secundário \\
Letras Financeiras do Tesouro- LFT & Cotação da NTN \\
Cálculo da cotação da LFT & Notas do Tesouro Nacional Série D- NTN-D \\
Cálculo do acréscimo ao valor de face da LFT & Leilão primário de NTN-D
\end{tabular}


No caso da obra de Vieira Sobrinho os temas LTN, NTN, LTF, taxa Selic e CDI constam no subtítulo da obra

No que se refere à títulos de renda fixa, a obra de Assaf Neto (2012) expõe, em seu sumário, um detalhamento com modalidades específicas e com variáveis que as afetam em particular, a exemplo da tributação. Tal detalhamento consta no quadro 5:

Quadro 5 - Exemplo de detalhamento de "Títulos de Renda Fixa" na obra de Assaf Neto

\begin{tabular}{|ll} 
Certificados/recibos de depósitos bancários & Obrigações (bônus) \\
CDB/RDB & Zero Coupon Bond \\
CDB/RDB com taxas prefixadas & Relação entre prazo de emissão e taxa de desconto \\
Taxa prefixada com rendimento final & com o valor do título \\
Extensões ao cálculo da taxa líquida & Títulos (bônus) com cupons \\
Taxa prefixada com rendimento periódico & Preço de mercado \\
CDB/RDB com taxas pós-fixadas & YieldtoMaturity- YTM \\
Confronto entre a taxa prefixada e a taxa pós-fixada & YTM e IRR \\
de juros & Relação entre valor do título e taxa de desconto \\
Desmembramento da taxa prefixada & Tributação vigente das aplicações de renda fixa \\
Diferentes variações dos índices de preços & Imposto de Renda (IR) \\
Custo de captação com recolhimento compulsório & Imposto sobre Operações Financeiras (IOF) \\
Debêntures &
\end{tabular}

Fonte: Elaborado a partir de Assaf Neto (2012)

O livro de Assaf Neto (2012) apresenta em seu sumário o tema "Avaliação de ações" organizado em dois eixos: "Aplicações em ações com prazo determinado" e "Aplicações em ações com prazo indeterminado". Deve-se mencionar que a implementação de Engenharia Financeira (HARTMAN; ENKE, 2007) nos cursos de graduação em Engenharia tem como requisito a familiaridade com modalidades de investimento cuja complexidade conceitual e pedagógica antecede os derivativos. Assim, a compreensão do universo de investimento das ações passa a ser condição necessária para que então se dê a compreensão (e mesmo criação) de instrumentos financeiros mais complexos. Quando os sumários dos livros analisados são confrontados com temas específicos constantes na matriz de conhecimentos pode-se identificar que as obras contemplam ampla maioria dos conteúdos, tal como expresso no quadro 6. 
Quadro 6 - Exemplos de conteúdos da matriz de conhecimentos da Resolução 1010/05 CONFEA explicitamente constantes nos sumários das três obras analisadas

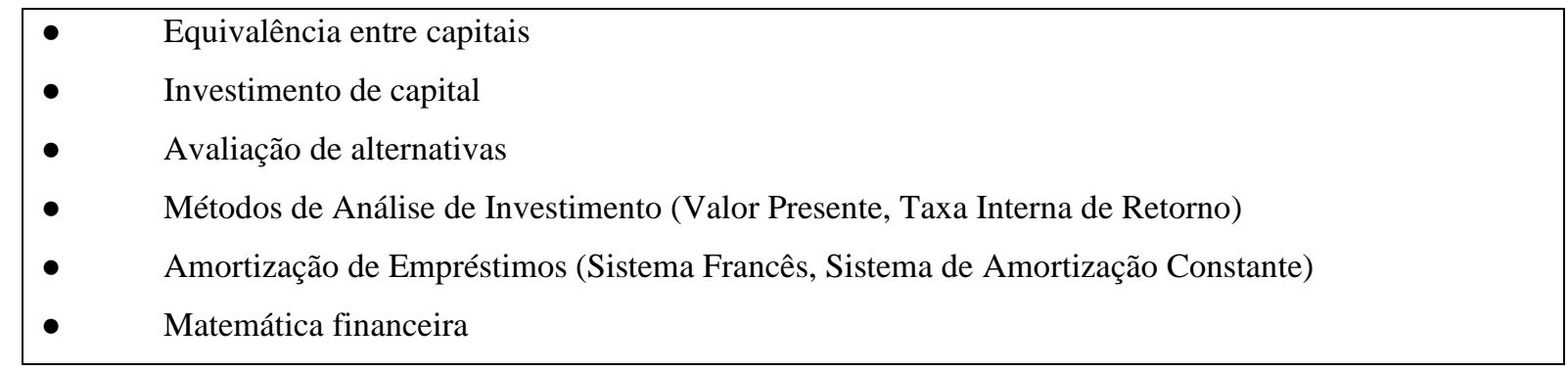

Fonte: Elaborado pelos autores a partir das análises (2020)

Temas como "investimento do projeto", "risco e incerteza", "conceituar e classificar projetos", ainda que não explicitados nestes termos nos sumários das obras comparadas devem estar sendo contemplados em capítulos sob diferentes denominações. No que se refere a temas como renda fixa e tributos pôde-se observar que não são temas explicitados no sumário de todas as obras. O tema "títulos", por sua vez, apareceu nas três obras apenas como "títulos públicos" e em duas delas como "títulos de renda fixa".

Cabe notar que ao proporcionar aos estudantes conhecimentos financeiros relacionados ao gerenciamento do capital de giro da organização, assim como conhecimentos sobre aplicações financeiras disponíveis para pessoa jurídica tem-se a possibilidade de formar profissionais aptos a aumentar a rentabilidade da organização e gerenciar riscos financeiros (incluindo hedge). Nesse sentido, a apresentação de instrumentos e modalidades de investimento tende a contribuir para a gestão do risco financeiro organizacional (LIZOTE; ANDRADE; SILVA; PEREIRA; PEREIRA, 2014. PINTO et al., 2019). Além disso, não se pode negligenciar o fato de que o acesso a tais conhecimentos também pode promover melhorias na gestão financeira pessoal dos estudantes, em seu próprio cotidiano, tal como argumentam Miranda e Philippsen (2014).

\section{Considerações finais}

Assumindo o pressuposto de que o livro didático ainda é um importante instrumento de ensino, este trabalho realizou uma análise comparativa dos temas contemplados em três livros de matemática financeira. Considerando a relevância da gestão financeira para a formação em Engenharia, o trabalho apresentou semelhanças e diferenças entre os conteúdos listados nos sumários de três livros de matemática financeira. Foram selecionados para análise os livros identificados como "mais vendidos" em distintos sites de vendas de livros. Dentre os 
resultados obtidos pode-se mencionar o fato de que as três obras comparadas contemplam temas elementares de matemática financeira (a exemplo de juros, capitalização, desconto e sistemas de amortização), enquanto temas como "análise de ações" são listados explicitamente no sumário de apenas uma das obras.

Tende em mente as discussões sobre o aprimoramento do ensino de finanças, deve-se ponderar que a inclusão de temas financeiros específicos no material didático pode contribuir para a formação de engenheiros capazes de realizar análises de investimentos eficazes e eficientes que se revertam em maior rentabilidade nas empresas. Além disso, a familiaridade com determinados instrumentos financeiros tende a contribuir para o próprio gerenciamento de risco organizacional.

Não obstante estejam presentes os avanços tecnológicos que proporcionam novas ferramentas educacionais, os livros didáticos ainda são ferramentas essenciais no processo de ensino, sendo, inclusive, itens que devem obrigatoriamente constar nas ementas de cursos de graduação no Brasil. Contudo, assumir que sejam eles a base instrumental da formação dos estudantes pode ser um equívoco.

Tendo este trabalho se limitado à comparação dos conteúdos explicitados no sumário dos livros, para trabalhos futuros julga-se relevante confrontar o conteúdo das obras com o constante nas ementas de disciplinas financeiras ministradas para cursos de Engenharia. Considerando-se que as obras analisadas, em figurando dentre as "Mais vendidas", contam com certo respaldo mercadológico de relevância, assume-se a hipótese de se tratar de obras didaticamente relevantes para o ensino de finanças. Complementarmente, faz-se pertinente acompanhar a evolução do conteúdo em relação a diferentes edições de uma mesma obra. Deve-se cogitar que, em razão do próprio preço da obra, edições mais recentes sejam preteridas em detrimento das mais antigas (potencialmente menos custosas).

Note-se que a seleção de obras didáticas tradicionalmente segue critérios definidos pelo docente da disciplina, sendo ele pautado por sua experiência e por seu perfil didático, por exemplo. Ademais, cabe ressaltar que as mudanças no mercado editorial, bem como as mudanças tecnológicas que afetam o ensino (que incluem recursos audiovisuais e e-books) não podem ser negligenciadas quando se procede a análise de livros didáticos.

\section{Referências}


ABEPRO - Associação Brasileira de Engenharia de Produção. Campo de atuação profissional da modalidade industrial: engenharia de produção. Disponível em: <http://www.abepro.org.br/arquivos/websites/1/Matriz\%20de\%20Conhecimento\%20$\% 20$ CREA's.pdf $>$ Acesso em 05/02/2019.

ASSAF NETO, Alexandre. Os métodos quantitativos de análise de investimentos. Caderno de Estudos, n. 6, out. 1992. Disponível em:

<http://www.scielo.br/scielo.php?script=sci_arttext\&pid=S1413-92511992000300001> Acesso em: 06 fev. 2020.

ASSAF NETO, Alexandre. Matemática financeira e suas aplicações. 12 ed. Atlas S.A: São Paulo, 2012.

CONFEA - Conselho Federal de Engenharia e Agrnonomia. Resolução n. 1.010 de 22 de agosto de 2005. Disponível em:

$<$ http://normativos.confea.org.br/ementas/visualiza.asp?idEmenta=550> Acesso em 06 fev. 2020.

EVANS, Emily; NACHTMANN, Heather; NEEDY, Kim A look into the engineering economy education. American Society for EngineeringEducationPaper, 2010. Disponível em:

<https://pdfs.semanticscholar.org/8da1/141190c897f72eb47e31010d907fe603b009.pdf?_ga= 2.124431433.2103233079.1581000934-42924372.1562152529> Acesso em 06 fev. 2020.

FARR, John. V.; MERINO, Donald. N., SCHAEFER, Charles, V.; Educating entry-level engineers: are broad-based business/managerial skills a key to sustaining the US innovationbased economy? International Journal of Engineering Education, v. 19, n. 2, p. 252-259, 2003.

HARTMAN, Joseph C; ENKE, David. Financial Engineering: the savior or end of engineering economy? Financial Engineering : The Savior or End of Engineering Economy? American Society for EngineeringEducationPaper, 2007. Disponível em: <https://pdfs.semanticscholar.org/9f80/90aeb79f411b75a34354f37d6e4797e60cfa.pdf?_ga=2. 64678124.2103233079.1581000934-42924372.1562152529> Acesso em: 06 fev. 2020.

IQBAL, Abdullah., FAROOQI, Nauman; SAUNDERS, Kent T. Teaching methods and assessment techniques used for the introductory level undergraduate finance courses in British and Irish universities. Journal of Economics and Finance Education, n. 5., v. 1, p. 47-61, 2006.

LELAND, Robert P. EnrichingtheEngineeringEconomics class. American Society for EngineeringEducationPaper, 2017. Disponível em:

<https://pdfs.semanticscholar.org/cc16/497df6d9088da78d1d981bb08116c106b413.pdf?_ga= 2.127526990.2103233079.1581000934-42924372.1562152529> Acesso em: 06 fev. 2020.

LIZOTE, Suzete Antonieta; ANDRADE, Debora Anderson; SILVA, Francini; PEREIRA, Rafael da Silva; PEREIRA, Willian da Silva. Análise de Investimentos: um Estudo Aplicado em uma Empresa do Ramo Alimentício. XI Simpósio de Excelência em Gestão e Tecnologia, 2014. Disponível em: <https://www.aedb.br/seget/arquivos/artigos 14/1220115.pdf> Acesso em 03 fev. 2020. 
MIRANDA, Lourdes Aparecida Nocette; PHILIPPSEN, Adriana Strieder. A importância da matemática financeira no cotidiano e na construção da cidadania. Os desafios da escola pública paranaense na perspectiva do professor PDE: artigos. Disponível em:

<http://www.diaadiaeducacao.pr.gov.br/portals/cadernospde/pdebusca/producoes_pde/2014/2 014_unespar-paranavai_mat_artigo_lourdes_aparecida_nocette.pdf > Acesso em: $06 \mathrm{fev}$. 2020 .

PEIXOTO, Alexandre Gomes. O uso de metodologias ativas como ferramenta de potencialização da aprendizagem de diagramas de caso de uso. Outras Palavras. v. 12, n. 2, p. 35-50, 2016.

PINTO, Marcos Moreira; OLINQUEVITCH, José Leônidas; MOROZINI, João Francisco; GUTH, Sérgio Cavagnoli; FASSINA, Paulo Henrique. Análise de viabilidade econômica de projetos de investimento: métodos utilizados em empresas fabricantes de balas do estado do Rio Grande do Sul. XXVI Congresso Brasileiro de Custos, 2019. Disponível em: <https://anaiscbc.emnuvens.com.br/anais/article/view/1812> Acesso em 06 fev. 2020.

SAMANEZ, Carlos Patrício. Matemática financeira. 5 ed. Pearson: São Paulo, 2010.

SANITÁ, Willian Roberto Valicelli; ZANATA, Manoela; CARETA, Bruna Luiza. Utilização de ferramentas logísticas online e gratuitas para desenvolvimento do aluno em práticas profissionalizantes aplicadas a metodologia ativa. Anais do VII Simpósio de Engenharia de Produção - SIMEP 2019. Disponível em: <https://www.even3.com.br/anais/7simep/141859utilizacao-de-ferramentas-logisticas-online-e-gratuitas-para-desenvolvimento-do-aluno-empraticas-profissionaliza/> Acesso em 06 fev. 2020.

VIEIRA SOBRINHO, José Dultra. Matemática Financeira - juros, capitalização simples e composta, sistemas de amortização Price e SAC, títulos públicos: LTN, NTN e LFT, taxas de Selic e CDI, utilização de calculadoras financeiras. 8 ed. Atlas: São Paulo, 2018.

ZOGHI, Shervin. Engineering Economics and its role in the engineering curricula. American Society for EngineeringEducationPaper, 2015. Disponível em:

<https://pdfs.semanticscholar.org/9f80/90aeb79f411b75a34354f37d6e4797e60cfa.pdf?_ga=2. 64678124.2103233079.1581000934-42924372.1562152529> Acesso em: 06 fev. 2020. 